\title{
Ultrafast Electronic Relaxation of Excited State of Biomimetic Metalloporphyrins in the Gas Phase
}

\author{
Niloufar Shafizadeh ${ }^{1}$, Minh-Huong Ha-Thi ${ }^{1}$, \\ Lionel Poisson ${ }^{2}$ and Benoit Soep ${ }^{2}$ \\ ${ }^{1}$ Institut des Sciences Moléculaires d'Orsay UMR 8214 CNRS, \\ Université de Paris-Sud 11, Bâtiment 210, Orsay, \\ ${ }^{2}$ Laboratoire Francis Perrin CEA/DSM/IRAMIS/SPAM - CNRS URA 2453, \\ CEA Saclay, Gif-sur-Yvette Cedex, \\ France
}

\section{Introduction}

Biomimetic molecules are systems that provide a direct and simpler reproduction of a molecular process essential to the living world, i.e. they mimic a function or a structure of a biomolecular edifice. The idea is to isolate this process and allow a selective study of a specific function by physicochemical methods, in a reductionist way. In that respect, gas phase studies of biomolecules are ideal, since they allow the application of a variety of highly sensitive and selective tools to the study of these model systems: mass spectrometry, photoelectron spectroscopy, for example, provided that the molecular system has been vaporised. In addition, the system is studied in absence of solvent, which greatly simplifies the interpretation of the fundamental effects and consequently allows to infer the effects of the medium since, in the gas phase solvents can be introduced in a gradual manner via clusters. Also gas phase measurements allow a direct comparison with results from quantum chemistry calculations. Great progresses have been fuelled in this direction by the developments of mass spectrometry. The studies in the gas phase have been mostly dedicated to the elucidation of the basic structure of the building blocks of proteins, i.e. small peptides (Simons, 2004).

In turn, other biological functions have been less studied by the gas phase biomimetic approach. Hematoproteins hosting hemoporphyrins (the heme, see fig 1) are ideal systems to examine in this way, since they are at the heart of the oxygen and small molecules transport within blood and that their active site is localised very precisely on the metal atom embedded in their central porphyrin. Thus, if one studies the active porphyrinic site of myoglobin at short time scales (in the subpicosecond time domain), the interaction with the environing protein (globin myoglobin) can be minimised.

In hemoproteins, the attachment of oxygen to the iron of the heme is a complex process involving a potential barrier. This barrier results from the crossing of two surfaces of 
different electronic and spin configuration of the metal atom (Harvey, 2000). Also, the metalligand bond is very sensitive and affected by charge flow in either direction, metal to porphyrin or the reverse, due to their similar ionisation energies. As an example, oxygen is bound to FeII but not to FeIII hemoproteins. Therefore, the study of the reverse process to ligandation, the dissociation of the ligand from the metal centre, is in practice only performed via electronic excitation through allowed electronic transitions of the heme porphyrin, providing sufficient energy to break the metal ligand bond. In these conditions, to be effected, this dissociation must involve first a reorganisation of the electron distribution between the porphyrin and the metal atom, Fe, i.e. an electronic relaxation. The electronic relaxation by itself does not necessitate a protein to be characterized, nor a ligand on the active metalloporphyrin. It is thus of great importance to investigate the electronic relaxation in protein-free metalloporphyrins and to track charge transfer states as intermediates in the dissociation of ligated porphyrins. The complete relaxation process and dissociation is extremely rapid from the $\mathrm{Q}$ band excited low spin Myoglobin-CO in the liquid phase; it is deemed to lead in less then $150 \mathrm{fs}$ to the ground state high spin Myoglobin (Fuss et al., 2004).

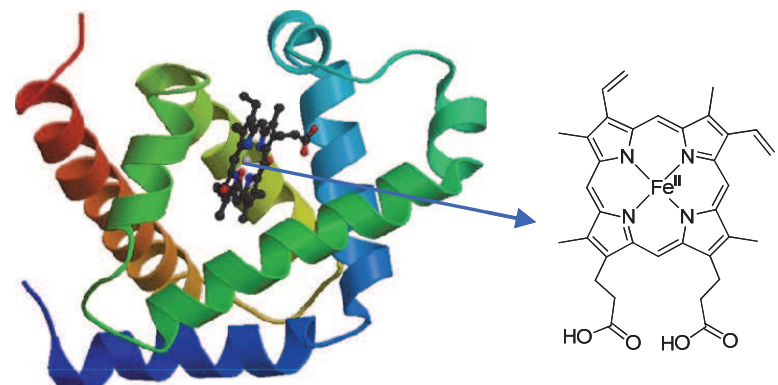

Fig. 1. Schematic picture of myoblobin and the active site, protoporphyrin IX (right).

Electron transfer and proton transfer are the most common mechanisms at the heart of many biochemical reactions. Zwitterions corresponding to the charge separation within a single molecule are commonly observed, as in soluble aminoacids (glycine $\mathrm{NH}_{3}{ }^{+} \mathrm{CH}_{2} \mathrm{COO}-$ ) and small protein strands, while phosphate ionic groups are ubiquitous in biochemical structures. On the other hand, excited state charge transfer (CT) processes are just as important. If one considers photosynthesis, there is a chain of excited CT transfers initiated within the chlorophylls in the reaction centres. This mechanism is far more complex to disentangle, due to its multiple pathways and brevity. In addition, charges flow, in this process between different subunits of the reaction centre. On the other hand, charge transfer processes in excited states can drive other processes, such as electronic relaxation. They are also difficult to characterise experimentally (Shafizadeh et al., 2009) and by quantum chemistry. In model nickel porphyrins, recent TDDFT calculations have shown the existence of a diversity of charge transfer states (CT), either porphyrin to metal or the reverse. In this nickel porphyrin (Patchkovskii et al., 2004), the lowest CT state is of the porphyrin to metal transfer type and can retain transient electronic excitation. On the experimental side, great endeavour has been put in solutions of hemoproteins and porphyrins to characterise by spectroscopic methods these CT states (Rodriguez et al., 1989). These studies show in general that the charge transfer is only partial and is a convenient way to picture the charge flow in these systems. 
Here, the ultrafast relaxation in metalloporphyrins is analysed in order to characterise the driving forces connecting the excited states of hemoporphyrins. It is showed that the mechanism is triggered by an ultrafast initial electron transfer from the porphyrin to the central metal by a comparative study of many systems. We have thus studied metalloporphyrins of increasing complexity from model tetraphenyl porphyrins hosting several transition metals, as biological systems host various metals within their central core, $\mathrm{Fe}, \mathrm{Cu}, \mathrm{Co}, \mathrm{Mg}$ (Milgrom, 1997). Based on this relaxation mechanism, we describe that of more complex biomimetic ones: Fe protoporphyrin IX (heme) and vitamin B12.

\section{Photophysics of porphyrin systems}

The continuous absorption spectra of heme and other metalloporphyrins observed in solutions are dominated by the intense B (Soret) and $\mathrm{Q}$ absorption bands involving excitation of the porphyrin cycle of the heme in a ${ }^{1}\left(\pi, \pi^{*}\right)$ transition (Gouterman, 1978). Aside these strong bands, at the edge of infra red, there are other less prominent bands of interest. These have been assigned by spectroscopic investigations and Stark spectroscopy to include porphyrin-to-metal charge transfer states CT and $(\mathrm{d}, \mathrm{d})$ electronic transitions (Eaton et al., 1978; Franzen et al., 1999).

The transients after a short pulse excitation have been abundantly (Steer and Tripathy, 2007) studied in solutions by transient absorption methods. However, the dynamics of relaxation of hemoporphyrins and other metalloporphyrins is complex and is not yet completely understood. The relaxation of simpler metalloporphyrins has been also studied extensively, by transient absorption measurements as models, especially the decay of nickel porphyrins. The relaxation of the $S_{1}$ state was characterized as a rapid transfer of excitation ( $\tau>350 \mathrm{fs}$ ) from the ring excited state $1\left(\pi, \pi^{*}\right)$ to a $\left(d, d^{*}\right)$ metal excited state. It was also observed that the $\left(\mathrm{d}, \mathrm{d}^{*}\right)$ state further relaxed to the ground state within $20 \mathrm{ps}$, probably due to the interaction with the solvent. On the other hand, there are few studies observing the decay of the $S_{2}$ excited state. For NiTPP excited in $S_{2}$, it has been inferred by the observation of broad transient spectra, that decay from $S_{2}$ to $S_{1}$ is the initial step to relaxation populating the $\left(d, d^{*}\right)$ states at times below $700 \mathrm{fs}$ (Rodriguez and Holten, 1989).

We reexamine here these conclusions and find a different interpretation, owing to the precision (energetic, temporal) offered by gas phase measurements and the absence of vibrational relaxation. On the other hand, Fe Protoporphyrin IX or heme, has been shown to follows a different relaxation pathways: Ishizaka et al. (Ishizaka et al., 2009) have studied the dynamics of relaxation of excited oxymyoglobin $\mathrm{Mb} \mathrm{O}_{2}\left(\mathrm{Fe}^{\mathrm{II}}\right)$ and met-myoglobin, met $\mathrm{Mb}$ (FeII) in solution. The authors, find that the fast relaxation is due to a rapid (femtosecond time scale) charge transfer from the porphyrin to the vacant $d$ orbital of the iron and the oxygen orbital is not involved in these transitions. These results suggest that the charge transfer from the porphyrin to the metal is the key event in excited state relaxation of the heme in $\mathrm{MbO} 2$.

In the present work, the relaxation dynamics of metalloporphyrin-based systems have been investigated by time dependent pump-probe spectroscopy, in the gas phase. The gas phase allows to measure the intrinsic properties of the active site, especially it separates the electronic and vibrational contributions in the relaxation process, and the absence of medium eliminates the vibrational cooling. This relaxation is found in transient absorption measurements as an additional transient, not present here. Time dependent spectroscopy can probe very fast relaxation processes immediately after excitation with a resolution 
determined only by the autocorrelation time of the pump and probe pulses. Electronic relaxation, resulting from non adiabatic couplings between the electronic states involved, is effected via a wavepacket motion after the ultrafast excitation (Mestdagh et al., 2000). The wavepacket goes through the funnels of the conical intersections into the surfaces of lower electronic states. The characterization of this wavepacket movement can give information on the dynamics of the excited system under study. This is achieved in pump probe time dependent experiments.

\subsection{Femtosecond pump-probe experiments}

The principle of the experiment is shown schematically in Fig 2. The initial state $\left(S_{2}\right)$ is populated by a $400 \mathrm{~nm}$ pulse laser (pump laser) and is ionised by a second laser pulse (probe laser). The delay between the pump and probe pulses is varied in a stepwise manner and $t=0$ is defined by the temporal coincidence of both pulses. Ionisation forms a molecular ion and an electron. In our setup, it is possible to detect either the ion or the electron. Detecting the ion leads to information on masses and fragments. Detecting the electron leads to information on the kinetic energy of the ejected electrons, reflecting the evolution of electronically excited intermediate states. In a simplified view of the conservation of momentum (vibrational) between the evolving state and the ion, the energy of ionisation is only dependent upon the electronic energy of the states probed. Thus as electronic energy is transformed into vibrational energy, the energy of ionisation increases (then, the photoelectron energy decreases, see figure 6 a). Thus, varying the time delay between pump and probe lasers, one can follow the evolution of the initial state, through various electronic states via their photoelectron energy (electron detection) or their ionisation efficiency (ion detection).

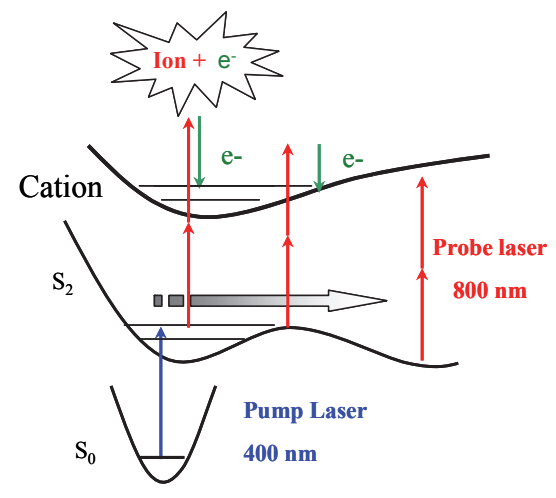

Fig. 2. Principle of a pump-probe experiment with ion or electron detection.

The experimental apparatus is a pulsed supersonic molecular beam coupled with a velocity map imaging device along the design of (Eppink and Parker, 1997) and a standard time-offlight mass spectrometer (TOF-MS). A schematic overview of experimental setup is shown in figure 3.

A carrier gas (helium) expanded supersonically through a pulse valve entrains the vapour of the molecule under study. The resulting molecular beam is collimated by a single skimmer before entering the interaction region. In this region, the neutral molecular beam is 
irradiated with the pump and probe lasers. In accordance with typical absorption spectra of porphyrin systems, the second harmonic of the Ti:sapphire femtosecond laser at $400 \mathrm{~nm}(70$ fs), was used as the pump pulse. The evolution of porphyrin excited in the Soret band $\left(\mathrm{S}_{2}\right)$ is followed by a second femtosecond laser, the probe laser. The probe laser at $800 \mathrm{~nm}$ passes through an optical delay line. This probe laser ionizes the excited system during its evolution. Thus, the generated ions and electrons can be followed temporally by varying the delay between the pump and probe lasers.

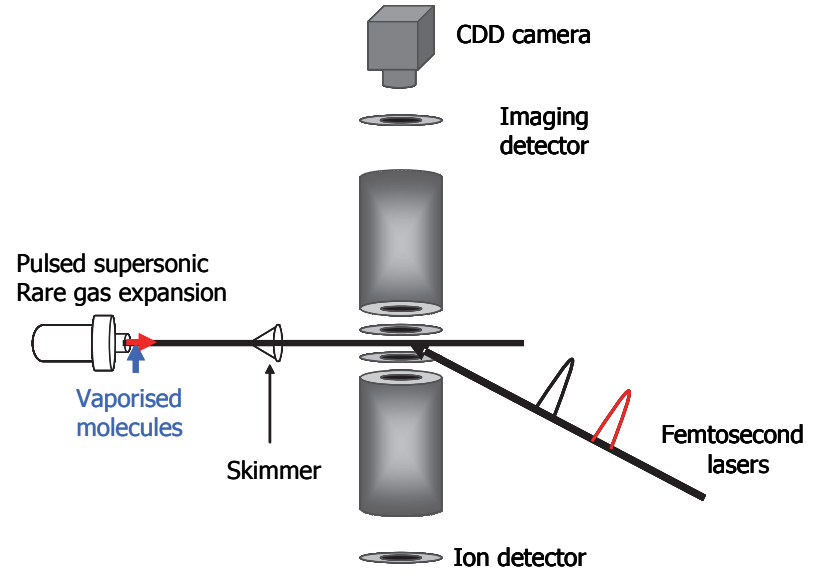

Fig. 3. Experimental setup.

\subsection{Evaporation methods}

The difficulty in performing the spectroscopy in the gas phase of molecules of biological interest is the vaporization of these systems. Most biomolecules are non volatile at room temperature and thermally unstable at vaporization. In order to minimize the fragmentation and maximize the number density of the species, different methods have been devised, minimising the heating or the duration of the heating. Traditional methods, such as electrospray, widely used in mass spectroscopy cannot be employed here since they produce mostly multiply charged or protonated molecules. For spectroscopic investigations of cold neutral systems, we used here two different methods: thermodesorption and laser vaporization coupled with a supersonic expansion. We will see that among the different molecules studied, some families are very stable towards heating, thus they can be simply heated until a sufficiently high vapour pressure is reached for the experiment, without any decomposition. On the other hand, more fragile systems, such as hemin or heme or vitamin $\mathrm{B}_{12}$ are totally fragmented in the heating process and required more delicate vaporization methods. For these molecules, we have implemented a new evaporation system based on laser desorption.

\subsubsection{Thermodesorption}

The molecules under study (commercial products from Aldrich) are mixed with graphite powder and pressed in a hydraulic press under $\sim 5$ Ton to form a cylindrical pellet. The pellet is heated in an oven and the temperature is typically held at about $500 \mathrm{~K}$. Graphite has 
been used as a matrix material for studying fragile molecules, since it is unreactive and protects the heated compounds from oxidation. It is a good heat conductor, and does not vaporize until $1000^{\circ} \mathrm{C}$. Immediately after evaporation, the molecule vapour is picked-up by a supersonic expansion of helium or argon. The supersonic expansion allows the immediate cooling of the molecular systems after vaporization.

The main advantages of this method are (i) it is very simple to use and (ii) the molecular beam is stable. This method has been applied to the vaporisation of a series of metallotetraphenylporphyrins and octaethylporphyrins. These two families of porphyrins are very robust and do not need excessive heating $\left(\sim 250^{\circ} \mathrm{C}\right)$.

\subsubsection{Laser desorption}

While thermodesorption method is efficient for introducing robust species into a molecular beam, it can not be used for the study of thermally unstable molecules, as is the case of most bio-molecules. In order to avoid thermal degradation of fragile systems, a very rapid heating is performed by means of laser desorption method. A short, pulsed laser, irradiation allows a very fast evaporation before the thermal degradation of molecules of interest. Furthermore, since most of laser energy is absorbed by graphite matrix rather than biomolecules, the photochemical degradation of molecules can be minimized (Piuzzi et al., 2000). To do this, a mixed graphite-molecule pellet is rotated at the tip of the nozzle of a pulsed valve. It is laser heated by a frequency doubled of a pulsed ns Nd:YAG laser, at 532 $\mathrm{nm}$. The YAG spot is adjusted to match the orifice of the supersonic beam and changed in position, to take into account the consumption of the active material. The time delays for the valve opening and the firing of the desorption laser are controlled within a $10 \mu$ sange with a sub-microsecond precision, which allow a rapid cooling of evaporated molecules.

This evaporation method permits measurements for a large class of biomolecules, which cannot be studied by thermodesorption method. We shall compare here mass spectra of a thermally unstable bio-molecule, hemin, obtained by these two different vaporization methods, in Fig 4.

Hemin is the salt form of the heme group with a $\mathrm{Cl}$ axial ligand. This complex molecule is very fragile and its fragmentation pattern depends on the vaporization method. Figure 4 shows the comparison between the two mass spectra of hemin ionized by $400+800 \mathrm{~nm}$ laser pulses, obtained by using the thermo-desorption fig $4 \mathrm{a}$ or by laser desorption, fig $4 \mathrm{~b}$. In the spectrum obtained by thermodesorption, the parent ion is absent, when comparing with the mass spectrum obtained by laser ablation in figure $4 \mathrm{~b}$. This latter spectrum shows two main peaks at 651 and $616 \mathrm{amu}$, corresponding to the parent ion hemin [Fe PP-Cl] ${ }^{+}$and the fragment $[\mathrm{Fe} \mathrm{PP}]^{+}$. The next two intense peaks are respectively at 571 and $557 \mathrm{amu}$, corresponding to $[\mathrm{Fe} \mathrm{PP} \text { minus } \mathrm{COOH}]^{+}$and $\left[\mathrm{Fe} \mathrm{PP} \text { minus } \mathrm{CH}_{2} \mathrm{COOH}\right]^{+}$. The next four peaks are respectively at 512,498, 484 and 472 uam. The peak at 512 corresponds to [Fe PP minus $\left.\mathrm{CH}_{2}(\mathrm{COOH})_{2}\right]^{+}$and the difference of mass between these four peaks is $14-15 \mathrm{amu}$ corresponding to plucking the side $-\mathrm{CH}_{2}$ or $-\mathrm{CH}_{3}$ groups, off the (porphyrin) macrocycle. The fragmentation pattern observed in figure $4 \mathrm{~b}$ is similar to the fragmentation reported by (Jones et al. 1994), using laser desorption. The peaks at lighter masses than $600 \mathrm{amu}$ are broad and symmetric, while the peaks at 651 and 616 amu are narrow and asymmetric. The shape of the peaks around 651 and $616 \mathrm{amu}$ is well reproduced by the isotopic distribution of the carbon, iron and chlorine atoms convoluted with the instrumental function corresponding to a mass resolution of 500 . The observed difference in width between fragments is due to multiphoton photofragmentation processes, between the light fragments 
and the intact [Fe PP-Cl] and [Fe PP]. In difference, since hemin is the parent molecule, still intact after evaporation, the fact that the shape of the peak of the [Fe PP] fragment is the same as that of hemin, is a strong indication that the $\mathrm{Cl}$ loss does not proceed through the ionization but is a result of the vaporization.

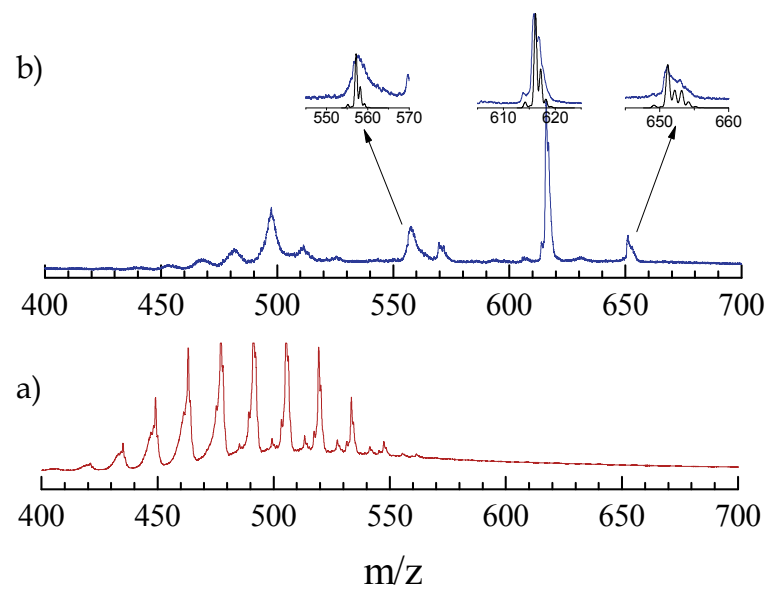

Fig. 4. Time of Flight mass spectra of hemin [FeIII PP-Cl] following 400/800 nm photoionization integrated over all pump/probe delays. (a) [ $\mathrm{Fe}$ III $\mathrm{PP}-\mathrm{Cl}]$ is evaporated using the thermodesorption technique $(\mathrm{b})\left[\mathrm{Fe}{ }^{\mathrm{III}} \mathrm{PP}-\mathrm{Cl}\right]$ is evaporated using the laser desorption technique. In the insert a simulation of the contours of the peaks at 651; 616 and 557 amu is obtained with the isotopic distribution of carbon iron and chlorine convoluted with the instrumental function corresponding to a mass resolution of 500 .

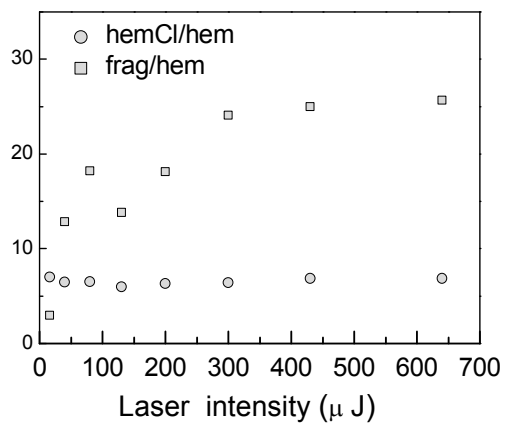

Fig. 5. Ratio of intensities of $\left[\mathrm{Fe}^{\mathrm{III}} \mathrm{PP}-\mathrm{Cl}\right]^{+}$to $\left[\mathrm{Fe}\right.$ II PP] ${ }^{+}$. The circles, $\circ$, represent the ratio of the area under the $[\mathrm{Fe} \text { III PP-Cl }]^{+}$mass peak to the area under the $\left[\mathrm{Fe}{ }^{I I} \mathrm{PP}\right]^{+}$peak. The squares, $\square$, represent the ratio of the area under the fragments of $[\mathrm{Fe} P P]^{+}$to the area of the $[\mathrm{Fe} P \mathrm{PP}]^{+}$peak. The $\mathrm{x}$ axis is the laser energy in $\mu \mathrm{J}$. 
To verify this, we have varied the intensity of one colour ionization with $400 \mathrm{~nm}$ pulses. Fig 5 shows the ratio of the area under the hemin peak to the area under the [Fe PP] peak. This ratio is almost constant within experimental fluctuations, while varying the intensity of the ionisation laser over the broad range $16 \mu \mathrm{J}-650 \mu \mathrm{J}$. This can be ascribed to the ionisation of [Fe PP-Cl] and [Fe PP] with the same efficiency by three $400 \mathrm{~nm}$ photons. In difference, the ratio of the areas under other fragments (masses between $420 \mathrm{amu}$ and $589 \mathrm{amu}$ ) peaks to the area of hemin, constantly increases while the laser intensity is increased; for the highest laser energies, this ratio goes to saturation.

We conclude that $[\mathrm{Fe} \mathrm{PP}-\mathrm{Cl}]$ and $[\mathrm{Fe} \mathrm{PP}]$ are formed within the vaporization process. In difference, the peaks located between $600 \mathrm{amu}$ and $420 \mathrm{amu}$ increase strongly with the intensity of the ionising femtosecond laser. This observation, together with their large width below $600 \mathrm{amu}$, larger than the simulated isotopic distribution in figure $4 \mathrm{~b}$, indicates that these peaks result from ionic fragmentation by the absorption of multiple $400 \mathrm{~nm}$ photons in hemin. In such case, metastable ions are formed that decompose during their flight through the ionisation region providing a trailing edge in their time of flight.

It can be seen here that in the presence of thermally fragile bonds, the most efficient method to form neutral molecules in the gas phase is laser desorption. This method can be considered as relatively mild since it permits to observe the parent ion and fewer fragments as compared to the thermodesorption. However, the molecular beam is less stable, due to fluctuations of the laser intensity in the evaporation process, and the unavoidable inhomogeneities of graphite pellet.

\section{Ultrafast relaxation of metalloporphyrins: from model molecules to biomimetic systems}

A systematic study of the decay of an ensemble of model metalloporphyrins serves to delineate the electronic relaxation mechanism that will be applied to biomimetic porphyrinlike molecules.

\subsection{Porphyrin models}

Tetraphenyl- and octaethyl- porphyrin are among the most thermal and chemical stable porphyrin families. We characterised with these molecules the decay mechanism applied to the more complex parent biomolecules, which contain also a porphyrin cycle or its derivative. The study of such model systems thus provides a basis for interpretation of the evolution in the more complex real systems.

The relaxation dynamics of this family has been widely investigated in solution by different techniques such as transient absorption (Drain et al., 1998; Rodriguez et al., 1991a) upconversion fluorescence spectroscopy (Gurzadyan et al., 1998) or resonant Raman spectroscopy (Petrich and Martin, 1989; Kumble et al., 1995). In the gas phase, very few works have been done on the spectroscopy of porphyrins. As an example, (Even et al., 1982), investigated the fluorescence excitation and they estimated the lifetime of the $S_{2}$ excited state in the picosecond time domain.

\subsection{1 $\mathrm{S}_{2}-\mathrm{S}_{1}$ Internal conversion in zinc tetraphenyl porphyrin}

We shall describe here a systematic study of the time evolution of electronically excited transition metal porphyrins. A central point is that we differentiate the case of filled shell, $3 \mathrm{~d}^{10}$ metalloporphyrin, ZnTPP and porphyrins containing open $\mathrm{d}$ shell metals, in order to 
interpret the effect of empty $d$ orbital on the electronic relaxation of excited state metalloporphyrins.

The time-resolved photoelectron and photoion spectra have been investigated for ZnTPP (Sorgues et al., 2006). The photoelectron spectrum integrated over all angles and pump/probe delay is displayed in Fig. 6a. The photoelectron signals as a function of the delay between the pump and probe pulses are presented in Fig. $6 \mathrm{~b}$ and Fig. $6 \mathrm{c}$ for low energy $(0.2 \mathrm{eV})$ and high energy $(0.8 \mathrm{eV})$ electrons, respectively. The photoelectrons of different kinetic energy presented different relaxation behaviours. For high photoelectron energies, the decay can be described by a short time constant of about $600 \mathrm{fs}$. On the other hand, at low electron energies, the photoelectron can be analyzed by two time constants, a short rise time with the same time constant (600 fs) followed by a plateau.

The photoelectron spectra reflect the dynamics of the transient excited state before ionization. This measurement provides additional information compared to the photoion signal that integrates this information. For example, we can differentiate photoelectrons resulting from different electronic excited states, thus their evolution can be followed separately, which is not the case in using the ion detection.

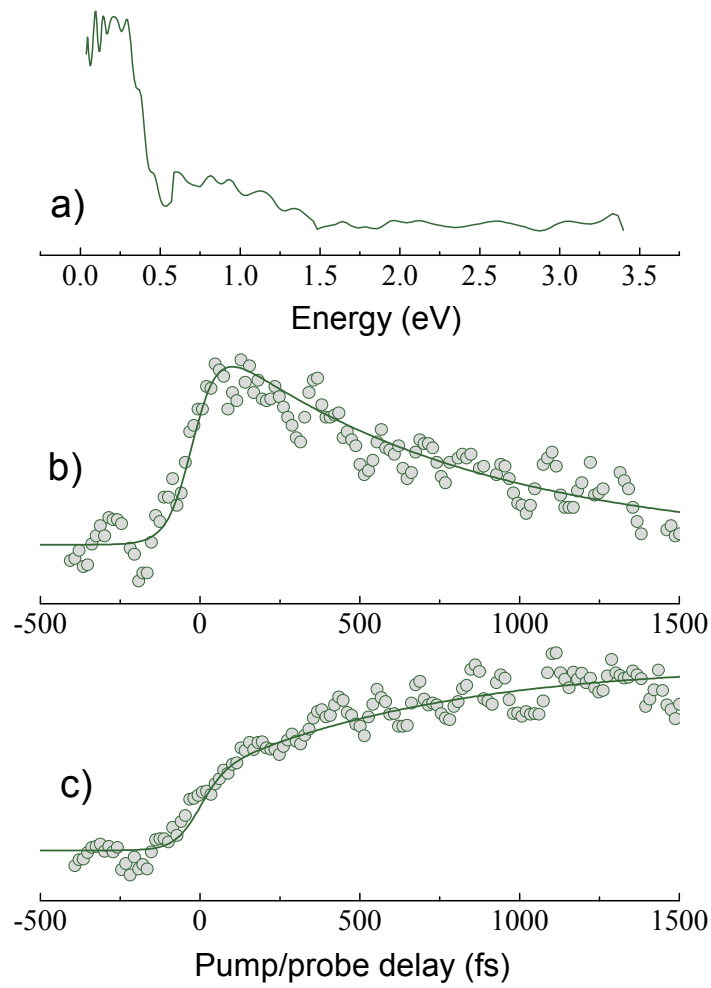

Fig. 6. a) Time integrated electron kinetic energy release (KER) spectrum of ZnTPP obtained under $(400 / 266 \mathrm{~nm})$ pump/probe conditions. Time evolution of the electron KER spectrum of ZnTPP b) at E=0.56 - $1.5 \mathrm{eV}$ and c ) at $\mathrm{E}=0.04-0.53 \mathrm{eV}$. 
The relaxation of the $S_{2}$ excited state of ZnTPP can be interpreted in Fig. 7. The first decay of higher kinetic energy photolectrons corresponds to the ionization of resonantly $S_{2}$ excited state with a $600 \mathrm{fs}$ lifetime. This decay corresponds to a rapid internal conversion from the $\mathrm{S}_{2}$ to the $S_{1}$ excited state. At lower kinetic energy, the photoelectron time resolved profiles picture the evolution of the $S_{1}$ excited state. This decay shows a rapid growth with the same time constant as the decay of $S_{2}$, suggesting that $S_{1}$ state is directly populated from the $S_{2}$ state. This result indicates that $S_{2}-S_{1}$ internal conversion is the main relaxation pathway of $S_{2}$ excited state of ZnTPP, which is in agreement with solution measurements of the low fluorescence quantum yield of $S_{2}$ excited state of porphyrins. The kinetics and plateau reached for the $S_{1}$ state matches the kinetics observed in solution, with $S_{1}$ fluorescent with a nanosecond lifetime (Liu et al., 2006). The above deactivation scheme is pictured in figure 7 with the $600 \mathrm{fs}$ time constant internal conversion connecting $S_{2}$ and $S_{1}$.

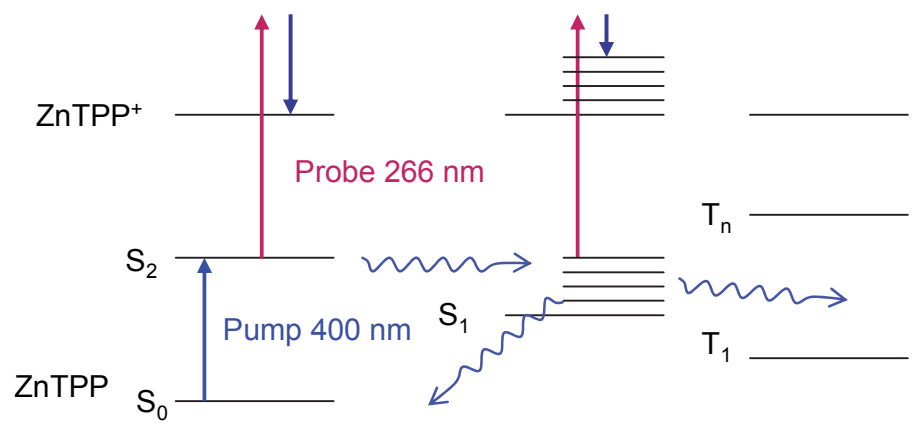

Fig. 7. Relaxation pathways of ZnTPP excited in the $S_{2}$ state.

To further assess the information provided by the photoelectron detection, the time resolved parent ion [ZnTPP] $]^{+}$signal is displayed in Fig. 8a. This decay can be simulated by two time constants, the first initial process of $600 \mathrm{fs}$, followed by a long-lived plateau, but here the rise of the $S_{1}$ state population is lost. This decay is the sum of the decay of the population of $S_{2}$ and the rise of $S_{1}$, the ionisation cross section of the latter one being lesser. These results show that the photoelectron and photoion detections are two complementary techniques to probe the dynamics of excited electronic states.

\subsubsection{Decay of $S_{2}$ in transition metal porphyrins}

We now examine the electronic relaxation of the $S_{2}$ state of a series of $3 \mathrm{~d}$-unfilled metal porphyrins (Sorgues et al. 2006). The time resolved ion signal of two porphyrins, CuOEP and NiOEP are presented in Fig. 8b and Fig. 8c, where the detection mass was centered at the parent ion mass. A similar relaxation profile was found for all the porphyrins hosting a $\mathrm{d}^{5}$ to $\mathrm{d}^{9}$ transition metal. The temporal evolution of these porphyrins consists of two exponential decay components. The results for the ensemble of metalloporphyrins studied are summarized in Table 1. As we can see in Table 1, a dramatic change has been observed for the lifetimes of porphyrins of 3d-unfilled metal as compared to ZnTPP. In fact, all the lifetimes of the $S_{2}$ excited states of these porphyrins are shorter by an order of magnitude. The first relaxation step occurs within the first $100 \mathrm{fs}$, while the second long-lived plateau of ZnTPP is replaced by a picosecond decay component. This observation indicates that these porphyrins of open $d$ shell metals exhibit a different relaxation pathway from that of ZnTPP. 


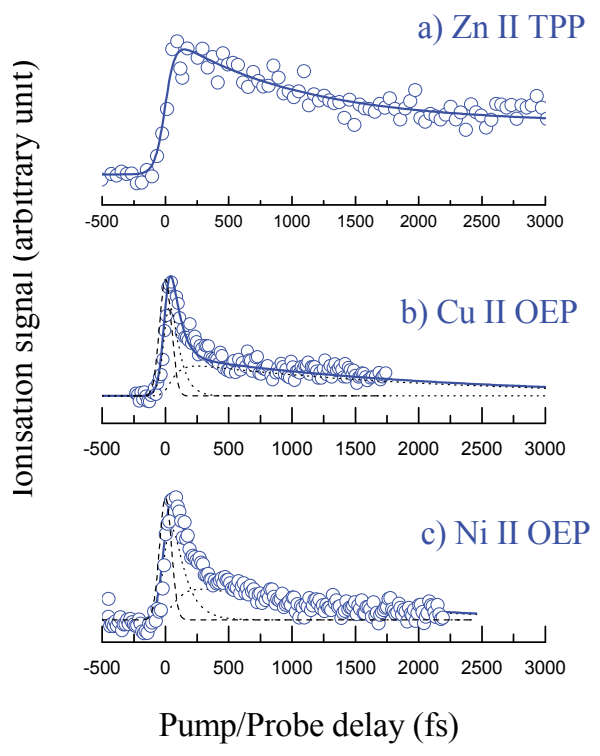

Fig. 8. Ion signals of (a) $[\mathrm{ZnTPP}]^{+}$, (b) $[\mathrm{CuOEP}]^{+}$and (c) $[\mathrm{NiOEP}]^{+}$as a function of time delay between the pump $(400 \mathrm{~nm})$ and the probe $(800 \mathrm{~nm})$

\begin{tabular}{ccccc}
\hline Metalloporphyrin & $\begin{array}{c}\text { Configuration } \\
\text { of the Metal }\end{array}$ & $\begin{array}{c}\text { Lifetime of } \\
\text { initial state } S_{2}\end{array}$ & $\begin{array}{c}\text { Lifetime of } \\
\text { relaxed state }\end{array}$ & $\sigma_{2} / \sigma_{1}$ \\
\hline Zn TPP & $\mathrm{d} 10$ & $600 \pm 50 \mathrm{fs}$ & $3 \mathrm{~ns}$ & $1.0 \pm 0.1$ \\
FeIII OEP & $\mathrm{d} 5$ & $50 \pm 20 \mathrm{fs}$ & $2000 \pm 200 \mathrm{fs}$ & $0.33 \pm 0.05$ \\
RuII OEP & $\mathrm{d} 6$ & $80 \pm 20 \mathrm{fs}$ & $1200 \pm 100 \mathrm{fs}$ & $0.33 \pm 0.05$ \\
RuII TPP & $\mathrm{d} 6$ & $70 \pm 20 \mathrm{fs}$ & $1200 \pm 100 \mathrm{fs}$ & $0.33 \pm 0.05$ \\
CoII TPP & $\mathrm{d} 7$ & $80 \pm 20 \mathrm{fs}$ & $500 \pm 100 \mathrm{fs}$ & $0.11 \pm 0.05$ \\
NiII TPP & $\mathrm{d} 8$ & $50 \pm 20 \mathrm{fs}$ & $375 \pm 50 \mathrm{fs}$ & $0.20 \pm 0.02$ \\
NiII OEP & $\mathrm{d} 8$ & $100 \pm 20 \mathrm{fs}$ & $1200 \pm 100 \mathrm{fs}$ & $0.25 \pm 0.02$ \\
CuII TPP & $\mathrm{d} 9$ & $65 \pm 20 \mathrm{fs}$ & $2000 \pm 200 \mathrm{fs}$ & $0.075 \pm 0.025$ \\
CuII OEP & $\mathrm{d} 9$ & $65 \pm 20 \mathrm{fs}$ & $2000 \pm 200 \mathrm{fs}$ & $0.25 \pm 0.02$ \\
\hline
\end{tabular}

Table 1. Decay lifetime of the S2 excited state of different metalloporphyrins: M-TPP or MOPE.

\subsubsection{The porphyrin-to-metal charge transfer model as driving mechanism for the relaxation of $\mathbf{S}_{2}$}

The nature of the ultrafast dynamics of these porphyrins containing $3 \mathrm{~d}$-unfilled metal is detailed in Fig. 9. As we showed in previous paragraph, the first 600 fs relaxation step of ZnTPP was attributed to the $S_{2}-S_{1}$ internal conversion. This channel can be excluded in the case of porphyrins of transition metals since this relaxation mechanism is centered on the porphyrin, its decay rate would depend only on the porphyrin macrocycle and not on the 
nature of the central metal. Indeed when comparing the evolution of an octaethyl and tetraphenyl transition metal porphyrin one finds no difference. Since there is such a difference between non transition metal $(\mathrm{Zn})$ and transition metal porphyrins, the mechanism must be ascribed to the coupling of the porphyrin with the metal.

The $S_{2}$ excited state is relaxed to a ring to metal charge transfer state, as we show now, owing to the presence of available empty $\mathrm{d}$ orbital of transition metal. This is the result of the following discussion. Once $S_{2}$ is excited, its population can, in principle, cascade through several electronic states lying between $S_{2}$ and the ground state via one of the following mechanisms:

i. Relaxation to the $S_{1}$ state.

ii. Relaxation to a charge ( $\overline{\mathrm{e}})$ transfer state, metal-to-porphyrin.

iii. Transfer of the excitation from the ring ${ }^{1}(\pi, \pi *)$ to a metal $\left(d, d^{*}\right)$ excited state .

iv. Relaxation to a triplet state located on the porphyrin ${ }^{3}(\pi, \pi *)$.

v. Relaxation to a ring-to-metal charge (ē) transfer state.

Channel i) was excluded above since the associated decay rate would depend only on the porphyrin modes and not on the nature of the central metal, Zn or transition metal. In this case the decay times would be similar for Zn and transition metals but they are found to be strikingly shorter for transition metals. In addition, photoelectron spectra of relaxed $\mathrm{Zn}$ porphyrin and transition metal porphyrins greatly differ, indicating a different relaxed excited state. The detected signal (ionisation efficiency) of the relaxed state, as a consequence, also differs as shown in Table 1.

Channel ii) is improbable since it does not occur for ZnTPP where Zn could transfer an electron to the porphyrin cycle. Also, strictly the same evolution is observed for FeII or FeIII protoporphyrins (see below). If a metal-to-porphyrin mechanism was involved, the withdrawal of an electron from Fe ${ }^{I I I}$ would be extremely improbable.

Channel iii) seems inefficient since it involves a double change in the electron configuration. Also it can be viewed as an excitation transfer from the porphyrin cycle to the metal through a Förster type mechanism (Förster, 1948). This dipole-dipole interaction process is highly improbable due to the bad overlap between the $\pi \pi^{*}$ excitation on the porphyrin and the $\mathrm{dd}^{*}$ forbidden transition of the metal.

Channel iv) where the presence of a metal flips the spin of the macrocycle electrons in a time less than $100 \mathrm{fs}$, seems highly improbable. However final triplet states after a longer evolution are likely triplet states as in copper prorphyrins.

Hence, by exclusion of the channels involving the porphyrin $i)$, the $\left(d, d^{*}\right)$ states of the metal iii), and the metal to porphyrin states ii), the most probable relaxation pathway from the $S_{2}$ excited state is the formation of a ring to metal charge transfer state v). In this case, the metal must have a vacant $d$ orbital with suitable energy, in order to accept the charge. This the case for transition metals up to copper, as pictured in figure 9. It is interesting to note that the addition of an electron acceptor group on the prophyrin ring is likely to channel electrons away from it. Rodriguez et al. (Rodriguez et al., 1991b) have observed the quenching of the fluorescence, hence the rapid decay of the $S_{1}$ state of the free base porphyrin substituted with a quinone group, which they correlate with a charge transfer from the porphyrinic cycle to the quinone. In addition, charge transfer is facile between the porphyrin and the metal and due to their respective low ionisation potential and high electron affinity. This porphyrin-to-metal CT mechanism is further supported by calculations on $\mathrm{Ni}$ porphyrin (NiTPP) (Patchkovski et al., 2004) where many low lying CT states are found, the lowest being of porphyrin-to-metal character. 


\subsubsection{Further evolution of the charge transfer state}

This rapid charge transfer (partial or complete) induces a direct mechanism through a conical intersection, because only a rapid wavepacket motion can connect the potential energy surfaces of $S_{2}$ and the charge transfer surface within this $100 \mathrm{fs}$ time domain. This charge transfer is followed by retrodonation to the porphyrins from one low lying $\mathrm{d}$ orbital to the empty $\pi$ orbital on the prophyrin, thus preparing a $\left(\mathrm{d}, \mathrm{d}^{*}\right)$ state localized on the metal (Fig.9). The further relaxation of $\left(\mathrm{d}, \mathrm{d}^{*}\right)$ state occurs in the 10 ps range which is relatively long lived in our detection conditions. In conclusion, the porphyrin-to-metal CT state is only a transient state that plays an important mediating role in the overall relaxation of these metalloporphyrins. On the other hand, if faster relaxation processes come at play, this relaxation mechanism can be superseded.

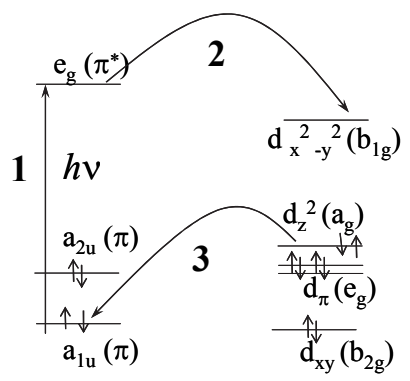

$$
\text { Porphyrin Metal -Ni(II) } \mathrm{d}^{8}
$$

Fig. 9. Mechanism of the relaxation pathway of the $S_{2}$ excited state of transition metals with empty d orbitals.

\subsection{Hemin: iron protoporphyrin IX}

We have shown in the previous paragraph that the time evolution of model porphyrins follows an initial charge transfer mechanism. Here, with the preceding model, the time evolution of gas phase excited of iron III protoporphyrin chloride, (hemin, FeIIIPPCl), and iron II protoporphyrin (heme FeIIPP), is analysed in the same terms and compared to the results of the zinc protoporphyrin. This latter system, owing to the lack of free $d$ orbitals on the metal site is unable to accept an electron at this location, as for the model ZnTPP.

The parent ion of hemin can only be observed in using laser desorption. In particular, our evaporation method allowed (serendipitously) detecting simultaneously FeIIPP and FeIIIPPCl, in a single experiment (Ha-Thi et al., 2010) .

The time resolved ion signals of hemin $\mathrm{Fe}$ IIIPPCl and heme FeIIPP are represented in Fig. 10a and Fig. 10b, respectively. The time evolution of these two molecules is very similar and can be analyzed by a three step sequential deactivation process after excitation. The ultrafast initial decay on the order of $50 \mathrm{fs}$ is followed by a second relaxation process of $250 \mathrm{fs}$.

According to the relaxation mechanism derived for the transition metal porphyrin models, a first transient is formed, a CT state, within $50 \mathrm{fs}$. The model mechanism applies well here since both hemin and heme undergo the same transient behaviour, i.e. through the same porphyrin to metal transfer. The second decay component, $250 \mathrm{fs}$ can be undoubtedly attributed to the relaxation of this charge transfer state leading to the formation of a $\mathrm{dd}^{*}$ 

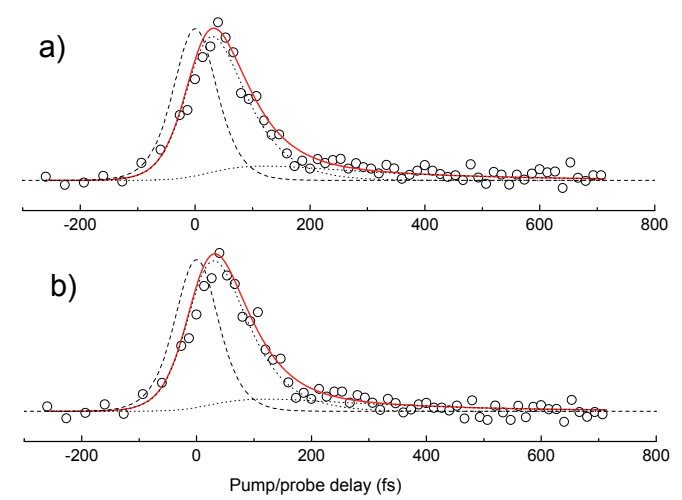

Fig. 10. Time evolution of hemin [Fe PP Cl], [Fe PP], while scanning the pump/probe delay $(400 / 800 \mathrm{~nm})(\mathrm{a})$ recorded at the mass of parent molecules [Fe PP Cl] $]^{+}$. (b) The observation is set at the mass of $[\mathrm{Fe} \mathrm{PP}]^{+}$. The experimental points are fitted by the solid line following a three level model. The contribution of each component is represented by dotted curves; the dashed curve indicates the cross correlation of the pump and probe pulse

excited state by a retrodonation of a metal electron of lower energy to the ring (see figure 9). In solutions, (Ishizaka et al., 2009), studying the myoglobin systems (FeMb), have concluded from the transient absorption spectra of $\mathrm{Fe}^{\mathrm{II}} \mathrm{MbO} 2$ and $\mathrm{Fe}$ IIImetMb, that the product detected at $600-700 \mathrm{~nm}$, is similar to the absorption band of the porphyrin cation. This is in accordance with a porphyrin-to-metal charge transfer into the available empty d orbital of iron, since only the porphyrin ${ }^{+}$ring has oscillator strength. Similarly, these authors observed a relaxation lifetime of $250 \mathrm{fs}$ for this charge transfer state.

The main difference in hemin with the previous model system is a new decay channel introduced by the porphyrin structure of hemin and heme: the competition that comes into play for the decay of the excited state $S_{2}$. It is seen, by studying $Z n P P$, that efficient $S_{2}-S_{1}$ internal conversion takes place and is extremely rapid (65 fs). The fact that the internal conversion and the deactivation via the charge transfer mechanism have a very similar relaxation rate means that the $65 \mathrm{fs}$ decay of $S_{2}$ in hemin and heme is probably a convolution of these two processes all leading to CT. However, the important information is the outcome of this relaxation since in a one electron picture, as showed in figure 9 , the excited $\mathrm{S}_{1}$ state is also of $\pi^{*}$ nature leading to the CT state, the route to the CT state being in this case less direct from $S_{2}$.

In conclusion, the charge transfer from the porphyrin to the vacant $d$ orbital at the transition metal site, is the key event in the relaxation for both compounds. This relaxation is very fast and is not affected by the presence of the chlorine atom, which remains spectator in the relaxation and is not dissociated from the heme group.

\subsection{Vitamin $\mathrm{B}_{12}$}

Here, we discuss the gas phase ultrafast electronic relaxation in another system, vitamin $B_{12}$, cyanocobalamine (figure 11) in light of the preceding mechanism. Vitamin $B_{12}$ is an essential human nutrient and plays a key role in the living cell, affecting DNA synthesis and regulation. In these processes the $\mathrm{CN}$ ligand to cobalt is exchanged for a methyl or hydroxyl group. The investigation of the photodissociation and the related electronic relaxation is examined here with the background of the previously mentioned systems. 


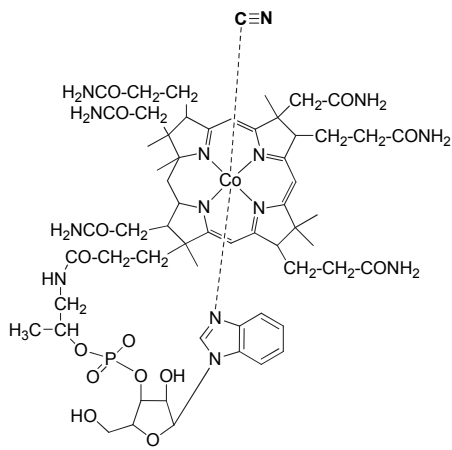

Fig. 11.Vitamine $\mathrm{B}_{12} \mathrm{C}_{63} \mathrm{H}_{88}$ Co $\mathrm{N}_{14} \mathrm{O}_{14} \mathrm{P}$.

The behaviour of the excited states of cyanocobalamine has been investigated in solution (Harris et al., 2007; Shiang et al., 2006). Recently we have reported, the first observation of the time evolution of vitamin $B_{12}$ in the gas phase (Shafizadeh et al., 2008). Vitamin $B_{12}$ is formed by a cobalt III atom coordinated in the pocket of a corrin. The corrin system is similar to the porphyrin ring except for one missing bridging methane $\left(\mathrm{CH}_{2}\right)$ and its partial saturation. The cobalt ion of the vitamin $\mathrm{B}_{12}$ is in a six-coordinate state, with four nitrogen atoms of the corrin ring and the two axial ligands, a cyano $(-\mathrm{CN})$ and a dimethylimidazole. The time evolution signal of vitamin $B_{12}$ obtained following an excitation at $400 \mathrm{~nm}$, is represented in Fig. 12a, detecting the parent ion. The vitamin $B_{12}$ signal is characterized by an ultrarapid $100 \pm 30$ fs decay, followed by a longer component, of picosecond time duration. This behaviour is viewed in comparison with the above mentioned CoTPP Fig $12 \mathrm{~b}$ (see paragraph 3.1.2...) which shows a very similar decay profile.
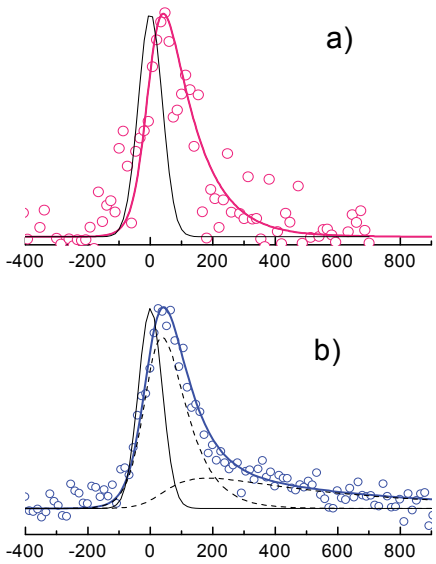

Fig. 12. Time evolution of vitamin $B_{12}$ (a), while scanning the pump/probe delay $(400 / 800$ $\mathrm{nm}$ ) compare to the time evolution of CoTPP (b). The experimental points are fitted by the solid line following a three level model. The contribution of each component is represented by dotted curves; the dashed curve indicates the cross correlation of the pump and probe pulse. 
As analysed for model metalloporphyrins, the initial decay of $B_{12}$ excited at $400 \mathrm{~nm}$ is assigned here to the relaxation to a porphyrin-to-metal charge transfer state, from a state that is likely $S_{2}$. However, the initial excited state is less well defined as in the case of more symmetric porphyrin molecules, like CoTPP. The subsequent picosecond decay can be interpreted in the same scheme as a retro-donation to the porphyrin, from a low d orbital of the metal. This second decay can be comparable with transient absorption measurements in condensed phase by Shiang et al. (Shiang et al., 2006).

Concerning the metal-ligand bond, excitation of $\mathrm{CN}$-ligated cobalt does not lead to the dissociation of $\mathrm{Co}-\mathrm{CN}$ bonding in the vitamin $\mathrm{B}_{12}$, consistently with the high affinity of the $\mathrm{CN}$ to the $\mathrm{Co}^{+}$metal ion.

\section{Conclusions}

A new experimental approach for the exploration of the electronic relaxation of fragile excited biomimetic molecules allows studying these systems in the gas phase without degradation.

Here, the ultrafast relaxation of the second electronically excited state of metalloporphyrins of $\pi \pi^{*}$ nature is analysed. It is showed that the mechanism is triggered by an ultrafast initial electron transfer from the porphyrin to the central metal by a comparative study of many systems. Metalloporhyrins of increasing complexity have been studied from model tetraphenyl porphyrins hosting several transition metals, to more complex biomimetic ones: Fe protoporphyrin IX (heme) and vitamin $\mathrm{B}_{12}$. The initial step of relaxation leads to an ultra fast porphyrin to metal charge transfer. This CT seems to be the doorway to the relaxation process of these compounds. In all systems studied here, the charge transfer direction is always from the porphyrin ring to metal. Once the CT state is reached, the time evolution varies from one species to other. In the case of nickel tetraphenyl porphyrins, the $(\mathrm{d}, \mathrm{d})$ state is reached. In the case of iron porphyrins the CT relaxes to the ground state as is the case for myoglobin (Kholodenko et al., 2000). The similarity between the solution and gas phase shows that the electronic relaxation properties of the heme group reflect that of hemoproteins and can serve as a biomimetic model.

This gas phase study paves the way for a series of investigations on ligated systems and possibly linked to small proteins. In this respect we have initiated the study in the gas phase of 'picket fence' porphyrins, i.e. models for the heme protein environment with simpler proteins, in order to study their time resolved photodissociation, the departure of the labile ligand.

\section{Acknowledgments}

The authors thank the technical staff of the femtosecond laser facility LUCA (Laser UltraCourt Accordable) of the CEA DSM/IRAMIS/SPAM who are responsible for developing, maintaining and running the femtosecond laser. The research described here has been supported by Triangle de la Physique under contract 2008-053T-SERPBIO

\section{References}

Drain, C. M., S. Gentemann, J. A. Roberts, N. Y. Nelson, C. J. Medforth, S. Jia, M. C. Simpson, K. M. Smith, J. Fajer, J. A. Shelnutt and D. Holten (1998). "Picosecond to 
Microsecond Photodynamics of a Nonplanar Nickel Porphyrin: Solvent Dielectric and Temperature Effects." Journal of the American Chemical Society 120(15): 3781.

Eaton, W. A., L. K. Hanson, P. J. Stephens, J. C. Sutherland and J. B. R. Dunn (1978). "Optical-Spectra of Oxy-Hemoglobin and Deoxyhemoglobin." Journal of the American Chemical Society 100(16): 4991-5003.

Eppink, A. and D. H. Parker (1997). "Velocity map imaging of ions and electrons using electrostatic lenses: Application in photoelectron and photofragment ion imaging of molecular oxygen." Review of Scientific Instruments 68(9): 3477-3484.

Even, U., J. Magen, J. Jortner, J. Friedman and H. Levanon (1982). "isolated ultracold porphyrins in supersonic expansion. I. Free base tetraphenylporphyrin and Zn." J.ournal of Chemical .Physics. 77: 4374-4383.

Förster, T. (1948). "Zwischenmolekulare Energiewanderung und Fluoreszenz." Annalen der Physik 437(1-2): 55-75.

Franzen, S., L. J. Moore, W. H. Woodruff and S. G. Boxer (1999). "Stark-Effect Spectroscopy of the Heme Charge-Transfer Bands of Deoxymyoglobin." Journal of Physical Chemistry B 103(16): 3070-3072.

Fuss, Champion, Blanchet, Nutt, Harvey, Soep, Buma, Blancafort, Martinez, Kohler, Temps, Doltsinis, Sundstrom, Koppel, Shalashilin, Child, Worth, Doltsinis, Rips, Olivucci, Truhlar, Baer, K. Balint, Yarkony, Miller, Whitaker, Brown, Stolow, Parker, Blanchet, Softley, Loomis and Hernandez (2004). "General discussion." Faraday Discussions 127: 227-266.

Gouterman, M. (1978). The Porphyrins, vol III. D. H. Dolphin. New-York, Academic Press. III: 1.

Gurzadyan, G. G., T. H. Tran-Thi and T. Gustavsson (1998). "Time-resolved fluorescence spectroscopy of high-lying electronic states of Zn-tetraphenylporphyrin." Journal of Chemical Physics 108(2): 385-388.

Ha-Thi, M. H., N. Shafizadeh, L. Poisson and B. Soep (2010). "First observation in the gas phase of the ultrafast electronic relaxation pathways of the S-2 states of heme and hemin." Physical Chemistry Chemical Physics 12(45): 14985-14993.

Harris, D. A., A. B. Stickrath, E. C. Carroll and R. J. Sension (2007). "Influence of environment on the electronic structure of Cob(III)alamins: Time-resolved absorption studies of the S-1 state spectrum and dynamics." Journal of the American Chemical Society 129(24): 7578-7585.

Harvey, J. N. (2000). "DFT computation of the intrinsic barrier to CO geminate recombination with heme compounds." Journal of the American Chemical Society 122(49): 12401-12402.

Ishizaka, S., T. Wada and N. Kitamura (2009). "Femtosecond transient absorption study on relaxation intermediates in oxymyoglobin." Photochemical $\mathcal{E}$ Photobiological Sciences 8(4): 562-566.

Jones, A. C., M. J. Dale, G. A. Keenan and P. R. R. Langridge-Smith (1994). Chemical Physics Letters 219: 174-180.

Kholodenko, Y., M. Volk, E. Gooding and R. M. Hochstrasser (2000). "Energy dissipation and relaxation process in deoxy myoglobin after photoexcitation in the soret region." Chemical Physics 259: 71-87.

Kumble, R., G. R. Loppnow, S. Z. Hu, A. Mukherjee, M. A. Thompson and T. G. Spiro (1995). "Studies of the Vibrational and Electronic-Structure of the S-1 Excited-States 
of Beta-Substituted Porphyrins by Picosecond Time-Resolved Resonance RamanSpectroscopy." Journal of Physical Chemistry 99(16): 5809-5816.

Liu, X., E. K. L. Yeow, S. Velate and R. P. Steer (2006). "Photophysics and spectroscopy of the higher electronic states of zinc metalloporphyrins: a theoretical and experimental study." Phys. Chem. Chem. Phys. 8: 1298 - 1309.

Mestdagh, J. M., J. P. Visticot, M. Elhanine and B. Soep (2000). "Prereactive evolution of monoalkenes excited in the $6 \mathrm{eV}$ region." Journal of Chemical Physics 113: 237-248.

Milgrom, L. R. (1997). The Colours of Life: An Introduction to the Chemistry of Porphyrins and Related Compounds. Oxford, Oxford University Press.

Patchkovskii, S., P. M. Kozlowski and M. Z. Zgierski (2004). "Theoretical analysis of singlet and triplet excited states of nickel porphyrins." The Journal of Chemical Physics 121(3): 1317-1324.

Petrich, J. W. and J. L. Martin (1989). "Ultrafast Absorption and Raman-Spectroscopy of Hemeproteins." Chemical Physics 131(1): 31-47.

Piuzzi, F., I. Dimicoli, M. Mons, B. Tardivel and Q. Zhao (2000). "A simple laser vaporization source for thermally fragile molecules coupled to a supersonic expansion: application to the spectroscopy of tryptophan." Chemical Physics Letters 320: 282-288.

Rodriguez, J. and D. Holten (1989). "Ultrafast vibrational dynamics of a photoexcited metalloporphyrin." The Journal of Chemical Physics 91(6): 3525-3531.

Rodriguez, J., C. Kirmaier and D. Holten (1989). "Optical-Properties of Metalloporphyrin Excited-States." Journal of the American Chemical Society 111(17): 6500-6506.

Rodriguez, J., C. Kirmaier and D. Holten (1991)a. "Time-Resolved and Static OpticalProperties of Vibrationally Excited Porphyrins." Journal of Chemical Physics 94(9): 6020-6029.

Rodriguez, J., C. Kirmaier, M. R. Johnson, R. A. Friesner, D. Holten and J. L. Sessler (1991)b. "Picosecond Studies of Quinone -Substitued Monometalated Porphyrin Dimers: Evidence for Superxchange-Mediated Electron Transfer in Photosynthetic Model System." Journal of the American Chemical Society 113: 1652-1659.

Shafizadeh, N., L. Poisson and B. Soep (2008). "Ultrafast electronic relaxation of excited state vitamin B-12 in the gas phase." Chemical Physics 350(1-3): 2-6.

Shafizadeh, N., B. Soep, J. M. Mestdagh and W. H. Breckenridge (2009). "Charge transfer in metal-atom-containing molecules in the gas phase." International Reviews in Physical Chemistry 28(3): 359-406.

Shiang, J. J., A. G. Cole, R. J. Sension, K. Hang, Y. Weng, J. S. Trommel, L. G. Marzilli and T. Lian (2006). "Ultrafast Excited-State Dynamics in Vitamin $B<s u b>12</$ sub $>$ and Related Cob(III)alamins." J. Am. Chem. Soc. 128: 801-808.

Simons J. P. (2004). "Bio-active molecules in the gas phase." Physical Chemistry Chemical Physics 6(10): E7-E7.

Sorgues, S., L. Poisson, K. Raffael, L. Krim, B. Soep and N. Shafizadeh (2006). "Femtosecond electronic relaxation of excited metalloporphyrins in the gas phase." Journal of Chemical Physics 124(11): 114302-10.

Steer, R. P. and U. Tripathy (2007). "The photophysics of metalloporphyrins excited in their Soret and higher energy UV absorption bands." Journal of Porphyrins and Phthalocyanines 11(4): 228. 


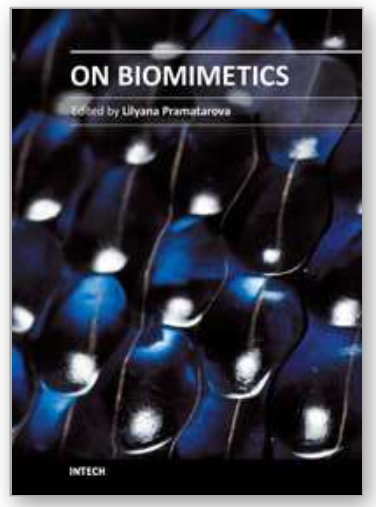

\author{
On Biomimetics \\ Edited by Dr. Lilyana Pramatarova
}

ISBN 978-953-307-271-5

Hard cover, 642 pages

Publisher InTech

Published online 29, August, 2011

Published in print edition August, 2011

Bio-mimicry is fundamental idea â€ How to mimic the Natureâ€TM by various methodologies as well as new ideas or suggestions on the creation of novel materials and functions. This book comprises seven sections on various perspectives of bio-mimicry in our life; Section 1 gives an overview of modeling of biomimetic materials; Section 2 presents a processing and design of biomaterials; Section 3 presents various aspects of design and application of biomimetic polymers and composites are discussed; Section 4 presents a general characterization of biomaterials; Section 5 proposes new examples for biomimetic systems; Section 6 summarizes chapters, concerning cells behavior through mimicry; Section 7 presents various applications of biomimetic materials are presented. Aimed at physicists, chemists and biologists interested in biomineralization, biochemistry, kinetics, solution chemistry. This book is also relevant to engineers and doctors interested in research and construction of biomimetic systems.

\title{
How to reference
}

In order to correctly reference this scholarly work, feel free to copy and paste the following:

Niloufar Shafizadeh, Minh-Huong Ha-Thi, Lionel Poisson and Benoit Soep (2011). Ultrafast Electronic Relaxation of Excited State of Biomimetic Metalloporphyrins in the Gas Phase, On Biomimetics, Dr. Lilyana Pramatarova (Ed.), ISBN: 978-953-307-271-5, InTech, Available from: http://www.intechopen.com/books/onbiomimetics/ultrafast-electronic-relaxation-of-excited-state-of-biomimetic-metalloporphyrins-in-the-gas-phase

\section{INTECH}

open science | open minds

\section{InTech Europe}

University Campus STeP Ri

Slavka Krautzeka 83/A

51000 Rijeka, Croatia

Phone: +385 (51) 770447

Fax: +385 (51) 686166

www.intechopen.com

\section{InTech China}

Unit 405, Office Block, Hotel Equatorial Shanghai No.65, Yan An Road (West), Shanghai, 200040, China 中国上海市延安西路65号上海国际贵都大饭店办公楼405单元 Phone: +86-21-62489820

Fax: $+86-21-62489821$ 
(C) 2011 The Author(s). Licensee IntechOpen. This chapter is distributed under the terms of the Creative Commons Attribution-NonCommercialShareAlike-3.0 License, which permits use, distribution and reproduction for non-commercial purposes, provided the original is properly cited and derivative works building on this content are distributed under the same license. 